\title{
Shear-Induced Amyloid Formation in the Brain: I. Potential Vascular and Parenchymal Processes
}

\author{
Conrad N. Trumbore* \\ Department of Chemistry, University of Delaware, Newark, Delaware, USA
}

Accepted 14 June 2016

\begin{abstract}
Shear distortion of amyloid-beta $(A \beta)$ solutions accelerates amyloid cascade reactions that may yield different toxic oligomers than those formed in quiescent solutions. Recent experiments indicate that cerebrospinal fluid (CSF) and interstitial fluid (ISF) containing $\mathrm{A} \beta$ flow through narrow brain perivascular pathways and brain parenchyma. This paper suggests that such flow causes shear distortion of $A \beta$ molecules involving conformation changes that may be one of the initiating events in the etiology of Alzheimer's disease. A $\beta$ shearing can occur in or around brain arteries and arterioles and is suggested as the origin of cerebral amyloid angiopathy deposits in cerebrovascular walls. Comparatively low flow rates of ISF within the narrow extracellular spaces (ECS) of the brain parenchyma are suggested as a possible initiating factor in both the formation of neurotoxic $A \beta_{42}$ oligomers and amyloid fibrils. $A \beta_{42}$ in slow-flowing ISF can gain significant shear energy at or near the walls of tortuous brain ECS flow paths, promoting the formation of a shear-distorted, excited state hydrophobic A $\beta_{42} *$ conformation. This $\mathrm{A} \beta_{42}$ * molecule could possibly be involved in one of two paths, one involving rapid adsorption to a brain membrane surface, ultimately forming neurotoxic oligomers on membranes, and the other ultimately forming plaque within the ECS flow pathways. Rising A $\beta$ concentrations combined with shear at or near critical brain membranes are proposed as contributing factors to Alzheimer's disease neurotoxicity. These hypotheses may be applicable in other neurodegenerative diseases, including tauopathies and alpha-synucleinopathies, in which shear-distorted proteins also may form in the brain ECS.
\end{abstract}

Keywords: Amyloid- $\beta$, cerebral amyloid angiopathy, cerebrospinal fluid, extracellular space, glymphatic system, interstitial fluid, protein conformation, protein misfolding

\section{INTRODUCTION}

\section{A perspective from amyloid chromatography}

A key biomarker for Alzheimer's disease (AD) progression is a decline in the concentration of the $1-42$ isoform of amyloid-beta $\left(A \beta_{42}\right)$ in patient cerebrospinal fluid (CSF), which is correlated with clinical progression of $\mathrm{AD}[1,2]$. The basis of this

\footnotetext{
*Correspondence to: Conrad N. Trumbore, 441 Crosslands Drive, Kennett Square, PA 19348, USA. Tel.: +1 610388 7093; Fax: +1 610388 5691; E-mail: conradt@udel.edu.
}

drop in $A \beta_{42}$ in CSF is believed to be sequestration of $A \beta_{42}$ into insoluble deposits within the brain tissue. This phenomenon, the decline in a soluble protein in its mobile phase resulting from aggregation, is reminiscent of a common occurrence encountered by protein biochemists when performing high performance liquid chromatography (HPLC) that uses stainless steel capillary connecting tubing.

Our research group used this HPLC connecting tubing in experiments that subjected solutions of a number of different aqueous protein solutions to liquid shear (defined below) by forcing these solutions through this very small inner diameter tubing [3]. 
Under certain experimental conditions, we demonstrated unexpected behavior, including deposition of solid protein aggregates on the capillary wall. We suggested that this deposition resulted from shearinduced aggregation of proteins due to the very small diameter of the capillary tubing.

Recently, it has been reported to this author [private communication, David Teplow] that HPLC amyloid studies in numerous laboratories find that significant amounts of amyloid disappear from the HPLC mobile phase. This result is anticipated by this author because of the many reports of sensitivity of $A \beta$, especially the $A \beta_{42}$ isoform, to shaking, stirring, and other shear-generating disturbances and especially because of the report in which $A \beta$ solutions are deliberately subjected to shear in carefully controlled circumstances and in which shear initiates a classic amyloid chemical cascade producing amyloid fibrils [4]. The author of this paper attributes these HPLC amyloid losses primarily to shear-induced amyloid wall deposition in HPLC capillary tubing [3]. Why is this wall deposition in narrow metal capillaries critical to $A D$ clinicians and researchers, and what is the suspected molecular explanation for this protein aggregation?

Experimental findings studying the aggregation behavior of $A \beta$ suggest that molecular shear, such as that encountered by proteins passing through HPLC capillaries, may contribute to the process of $\mathrm{A} \beta$ aggregation. Dustan and coworkers [4] have suggested that even gentle shear used in their experiments, where only one hydrogen bond per molecule is broken, can initiate an amyloid cascade producing $\mathrm{A} \beta$ solids. It is well established that $\mathrm{A} \beta$ aggregates form more readily in solutions undergoing sheargenerating disturbances. The question raised in this paper is whether similar shear forces are present in the brain that lead to $A \beta$ aggregation and other possible related detrimental health consequences involving $\mathrm{A} \beta$.

Recent studies describing the bulk flow of CSF and interstitial fluid (ISF) along perivascular pathways (PVP) suggest to this author that $A \beta$ in these solutions moving through tightly confined spaces are probably undergoing a similar type of shear stress to that seen in the shear experiments described above. The following discussion will suggest the influence that molecular shear may have on the aggregation of proteins like $A \beta$, detail how bulk flow through PVP and the wider brain extracellular compartment may initiate shear-induced chemical processes, especially in the presence of membranes, and propose a role for this process in the membrane adsorption and deposition of $A \beta_{42}$ in extracellular plaques and $A \beta_{40}$ in the walls of cerebral arteries in cerebral amyloid angiopathy (CAA).

\section{What is liquid shear?}

Figure 1 illustrates various types of shear. There are two important types of liquid shear [5] that can occur in the brain, laminar and extensional. Laminar shear is found, for example, in liquids confined to flow within a narrow bore capillary tube (Fig. 1c) where the laminar flow rate profile of the liquid is bullet-shaped, fastest in the center and zero at the capillary wall. Any water-based liquid moving parallel to a wetted capillary wall will incur a maximum liquid shear stress in that solid-liquid boundary capillary wall region, and the minimum shear is found in the very center of the capillary. Why is this so? We can consider a flowing liquid inside a capillary as a set of very thin, hollow, very long, telescoping concentric liquid cylinders of equal thickness. In laminar flow, each cylinder flows within the capillary at a different speed than its neighbor because the layer next to the wall is an aqueous solution that sticks to the wall and does not move. The largest speed difference between these thin neighboring cylinder layers is found at the wall and the smallest speed difference is found at the very center of the capillary. Liquid shear is defined as the speed difference between each layer and its neighbor. Therefore, the largest shear rate will be found at the wall where the flow rate of the wetting layer immediately next to the wall is zero. Thus the resulting maximum shear stress is found between the flowing liquid layer next to that solid wall liquid boundary. This is illustrated in Fig. 2, where the flow rate differences, indicated by the differences between neighboring arrow lengths, is smallest in the center and largest next to the wall, where there is no arrow at the wall because the flow rate there is zero. Figure 2 also illustrates the differing shear distortion effects on coiled polymer molecules residing in different parts of the capillary stream, the highest molecular distortion occurring next to the wall and the lowest in the center of the capillary. The molecule scale is greatly exaggerated in comparison with the scale of the capillary.

Extensional shear is generated, as illustrated in Fig. 1d, when liquid flows through a tube whose diameter decreases with distance down the flow direction of the tube. A macroscopic example of extensional shear is a stream with dangerous rapids, where the water is forced to flow between many large exposed 

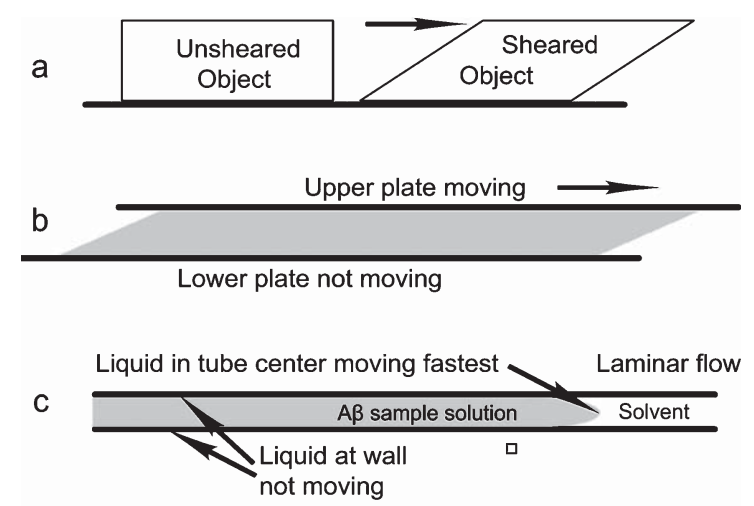

Flow from wide to narrow diameter tubing

d

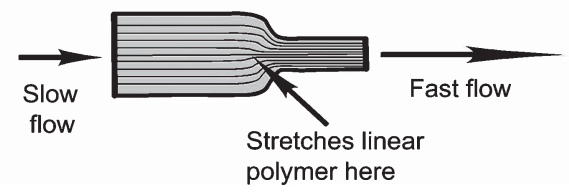

Fig. 1. Different types of shear (cross sections): (a) Sheared solid; (b) liquid between two plates, one stationary; (c) liquid flowing in a capillary or two stationary plates; (d) liquid flowing through tubes with different diameters. Laminar liquid shear is found in (b) and (c). Both laminar and extensional shear are found in (d).

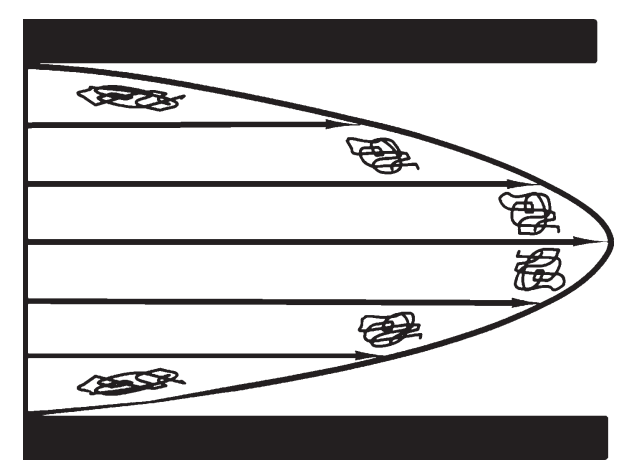

Fig. 2. Laminar shear on a macromolecule increases as the molecule approaches walls. Scale is greatly magnified for macromolecules. Length of arrows is proportional to flow rate. This figure illustrates either a cross section through a tube axis or liquid trapped between two flat plates. Note the difference between successive arrow lengths as one moves toward the center. This difference in layer flow velocity demonstrates that liquid shear and molecular distortion is greatest at the wall when there is laminar flow and at a minimum in the center region.

rocks. As water approaches the gap between two large rocks, it accelerates and then slows down again after passing between them, with eddies behind the rocks on the downstream side. If a coil of rope is put in upstream from the rocks, it has a tendency to stretch out as it transits the passage between the rocks. This is because there are accelerating water flow rates on

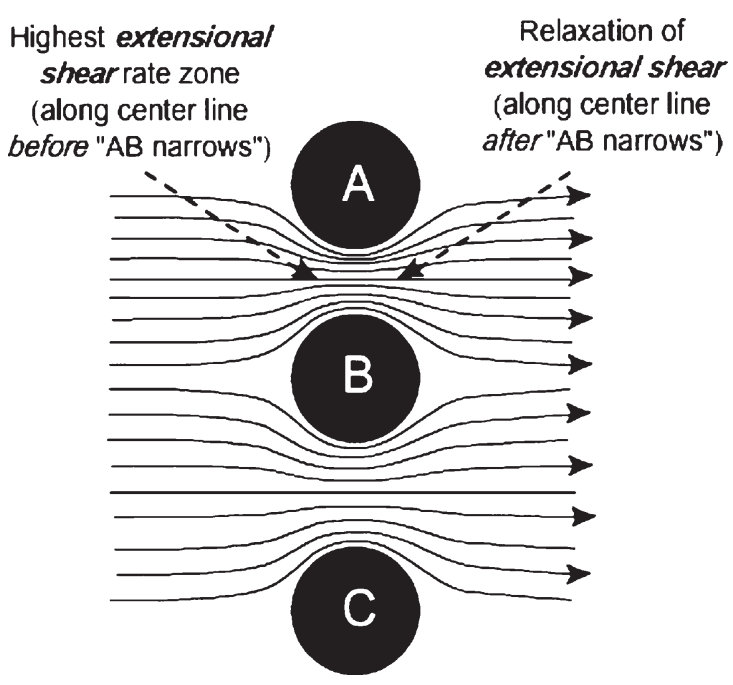

Fig. 3. Illustration of extensional shear, where a long molecule entering the restricted flow zone between flow obstructions A and $\mathrm{B}$ along the center line senses a faster flow at the center line between the objects than at any point to the left of that on the center line, thus stretching the molecule because of the difference in the flow rates. As the molecule exits the restricted flow zone the stretching force is relaxed.

the upstream side as the rope approaches the rock gap and decelerating water flow rates on the downstream side of the narrow rock passage, relaxing the stretch. Thus, initially the front part of the rope can experience stronger acceleration than the lagging part of the rope. The narrower the passage between the rocks, the faster the maximum flow through the passage between the rocks and the larger the extensional shear, illustrated in Fig. 3.

Extensional shear can stretch a flexible polymer molecule. This is especially true of a certain class of protein polymers designated as intrinsically disordered proteins (IDP) [6] that have many different possible conformations at normal temperatures rather than one fixed conformation. This IDP class includes $\mathrm{A} \beta$ molecules, among other amyloids.

This paper proposes that both laminar and extensional shear of $\mathrm{A} \beta$-containing CSF and ISF can play important roles in both the parenchymal and vascular parts of the brain by providing enough energy in the form of shear to induce molecular conformational changes that open new chemical pathways to flexible $A \beta$ and other IDP molecules. It is proposed that these very narrow, congested brain pathways can lead to both aggregation and interaction with membrane walls confining the CSF and ISF. 


\section{THE PROPOSED ROLE OF SHEAR IN AMYLOID-BETA AGGREGATION}

There are two major natural isoforms of $A \beta$, $A \beta 1-40\left(A \beta_{40}\right)$, and $A \beta 1-42\left(A \beta_{42}\right)$, composed of chains of 40 and 42 linked amino acids, respectively. Each of these isoforms, under certain temperature, $\mathrm{pH}$, and $\mathrm{A} \beta$ concentration, and solution preparation conditions, spontaneously undergoes an in vitro amyloid cascade aggregation, which involves noncovalent $\mathrm{A} \beta$ molecule bonding reactions that proceed through a low molecular mass oligomer stage, proceeding then to a protofibril, and then to a fibril state that is similar in molecular structure with that found in $\mathrm{AD}$ plaque [7]. Shaking and stirring of $A \beta$ solutions initiates $A \beta$ aggregation under conditions where no cascade reaction occurs in the absence of shear.

Studies of $\mathrm{A} \beta$ fibril formation have demonstrated that both the kinetics of fibril formation [8] and types of fibril conformations [7] are qualitatively different in quiescent and agitated solutions, implying that shear associated with agitation-or that associated with passage of restricted flow in narrow passages such as that which occurs in a narrow diameter capillary tube-may exert a powerful influence on $A \beta$ aggregation. Shear may therefore be a key variable in the formation of amyloid plaques if $\mathrm{A} \beta$-containing fluids are subject to shear within the brain.

$\mathrm{A} \beta_{42}$, being a more hydrophobic molecule [9], is much more sensitive to agitation-induced aggregation shear than $A \beta_{40}$. Experiments with $A \beta$ utilizing carefully defined shear conditions where there is not enough shear energy to break more than one hydrogen bond per $A \beta$ molecule, have demonstrated shearinduced formation of amyloid fibrils [4], indicating that it is not necessary to unfold the $A \beta$ molecule to initiate the $A \beta$ cascade reaction.

Given the above discussion, our capillary protein results, and the report of similar losses from $A \beta$ solutions undergoing HPLC experiments, this author suggests that these experimental results have a common shear-induced aggregation explanation that may provide clues to plaque formation in the brain [10]. For example, given the very high sensitivity of $A \beta_{42}$ to shear in comparison with the $A \beta_{40}$ isoform [8, $11]$, it might be expected that, in brain regions where brain fluids containing $A \beta$ are exposed to mild shear, $A \beta_{42}$ might be subject to aggregation, whereas $A \beta_{40}$ would be more resistant to shear. Thus, the CSF or ISF monomer $A \beta_{42} / A \beta_{40}$ concentration ratio would drop in the brain region with low shear as the aggregated $A \beta_{42}$ selectively dropped out of solution. This same drop in $A \beta_{42} / A \beta_{40}$ ratio in spinal tap CSF is currently used as one of a limited number of successful biomarkers for following the progression of AD.

The fluid flow geometry used in nearly all published in vitro $\mathrm{A} \beta$ shear experiments is generally not appropriate for modeling brain processes because the $A \beta$ fluids did not have the same degree of flow restrictions that lead to the types of shear, especially extensional shear, suspected to be present in the brain. The author suggests that our capillary experiments [3], updated and appropriately modified, can be used as a more appropriate model for studying shear processes such as ISF passage through the very narrow passages in brain parenchyma and CSF in vascular regions.

Relatively little is known about the fluid flow rates in those locations of the brain in which fluid shear might exist. It was earlier thought that there was no fluid flow within the brain parenchyma [12]. However, the work of Cserr and coworkers [13] appears to demonstrate that interstitial bulk flow does indeed take place. Evidence from studies carried out in rodents, including mice and rats, shows that CSF moves relatively rapidly along PVP surrounding brain blood vessels leading into the brain while ISF is reportedly drained along different periarterial pathways leading out of the brain [14]. It is claimed that both of these brain fluid flows take place through basement membranes, the ISF through muscle cell membranes and the CSF through pial-glial basement membranes [14].

\section{Shear in the brain vascular system}

Because of uncertainties in the structures and flow rates of CSF in the PVP, it is currently not possible to pinpoint the exact PVP structures or vascular regions where shear might be important. However, it is clear that $\mathrm{A} \beta$-containing CSF flows into the brain perivascular pathway close to the outer walls of the arterioles. Figure 4 illustrates the probable locations of these postulated PVP locations [14-16].

It was originally suggested that the flow of CSF through the PVP is powered only by cerebral arterial pulsation. However, reports of recent NMR investigations of CSF-containing human brain ventricles [17] suggest that the prime powering force for CSF flow in brain ventricles is the breath inhalation-exhalation cycle, with superimposed minor higher frequency heart pulse cycle contributions. These studies deal with CSF flow through the ventricular and cisternal 


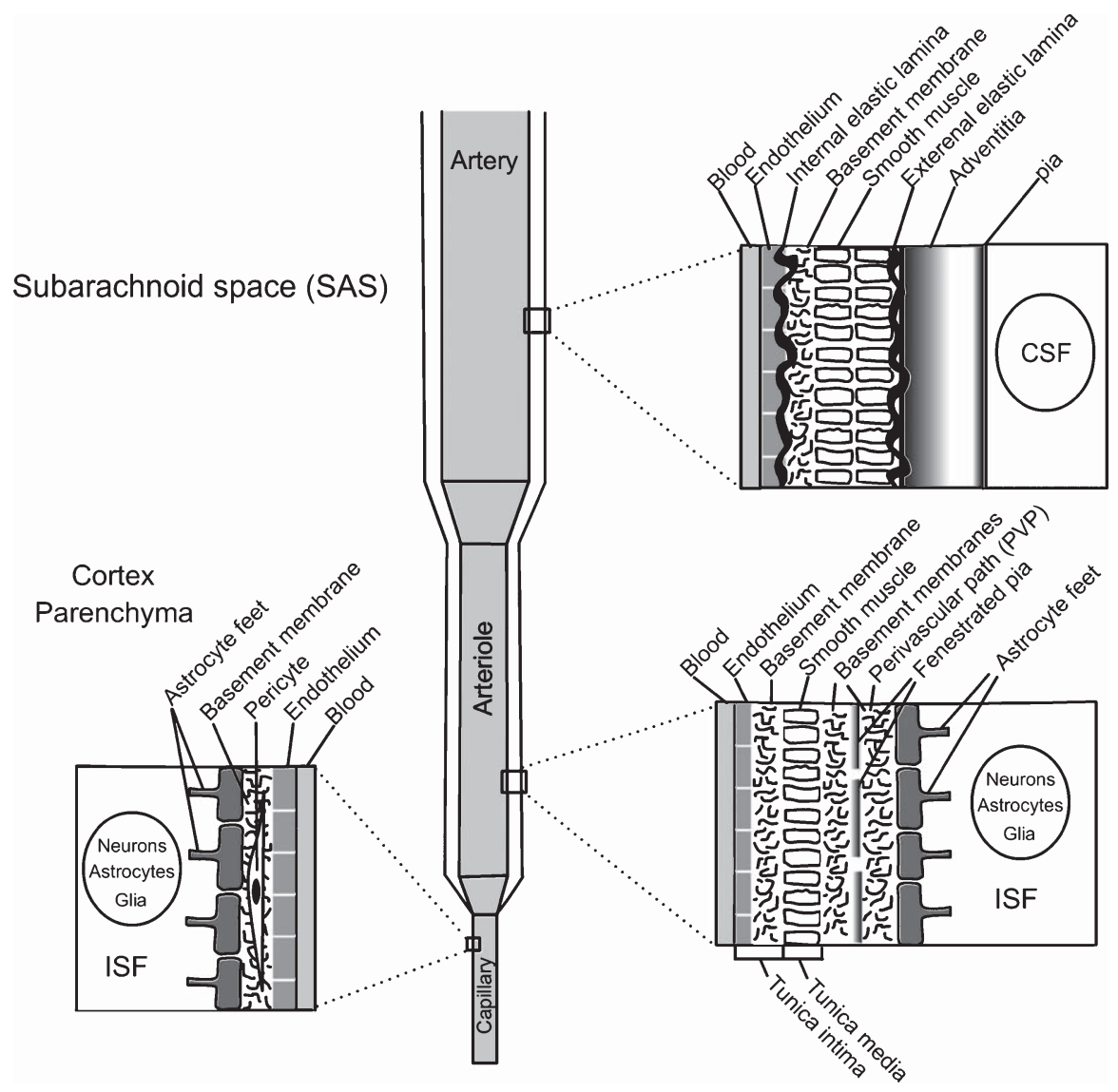

Fig. 4. Composition of the brain vascular arterial and capillary system. More detailed discussion on arteriole wall composition can be found in Morris et al. [14].

spaces, and do not address perivascular fluid movement. It is reasonable to presume that respiration plays an important role, since it is apparently a key driver of CSF flow, but this has not been tested to date.

Blood pressure and arterial blood flow rates are controlled mainly by arterioles, whose endothelial cells control by chemical and mechanical means about $80 \%$ of the flow rate through the arterioles and into the capillaries. The means by which this is accomplished are complex, but it is clear that such control involves continuous adjusting of large parts of the arteriolar wall [18]. It is not known how these adjustments affect the coupled flow and shear rates of the CSF in the PVP. Tracing CSF flow through the artery PVP to the narrower arteriolar region, it would be expected that the arteriolar PVP would also narrow because of overall space restrictions. Such narrowing could be a source of extensional shear in addition to laminar shear on walls confining the flowing CSF. If this flow is through basement membranes [14], then additional shear from membrane structures will be encountered, as discussed below.

Thus, periarterial $\mathrm{A} \beta$ molecules are subject to shear forces that can promote conformational changes that store shear energy in shear-distorted $\mathrm{A} \beta *$ molecules. These unstable molecules now are prone to thermodynamic forces that drive them to preferentially diffuse to regions of lower shear [19]. One such region is the overlapping endfoot processes (OEP) between astrocyte feet in the arteriolar PVP (see Fig. 4). This preferential migration would tend to increase the $A \beta$ concentration in OEP regions of the PVP. (See Supplementary Material A for further discussion of this concept.)

\section{ISF efflux routes used to rid brain parenchyma of $A \beta$ waste}

$A \beta$ is generated from the enzyme degradation of amyloid- $\beta$ protein precursor in the synapse membrane. However, $A \beta$ becomes part of a toxic 


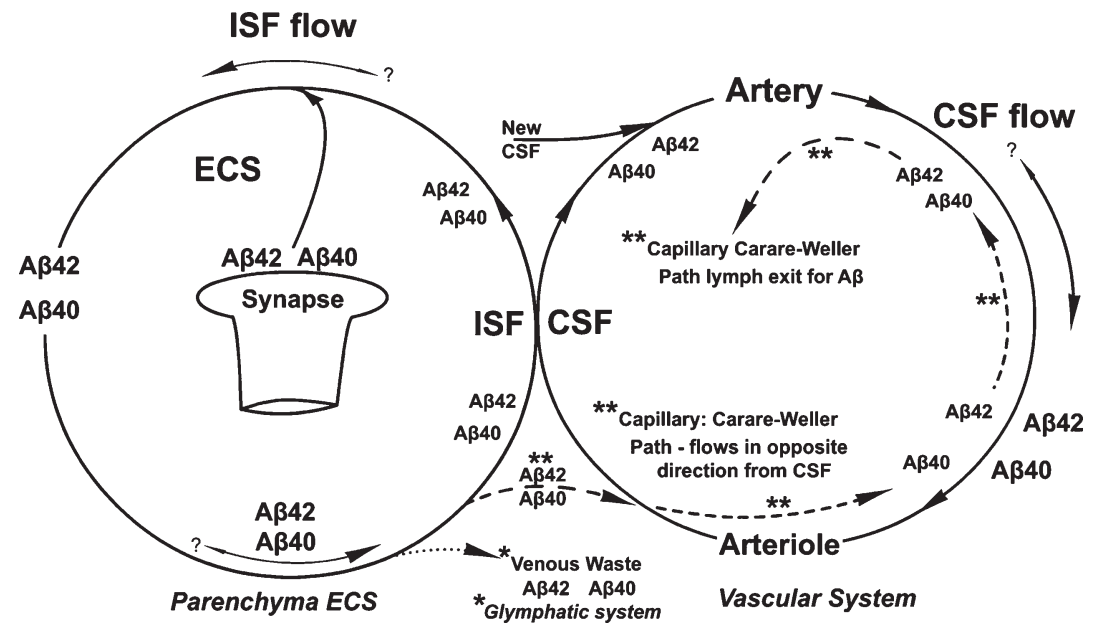

Fig. 5. An overview of the separate flow patterns of the ISF and CSF and their intersection in the absence of shear-induced A $\beta$ aggregation processes. Shown are the two different proposed pathways for the recycling of the two A $\beta$ isoforms, the proposed glymphatic (*) and the Carare-Weller perivascular (**) systems. No shear-processes are shown in this diagram.

molecule when incorporated into non-covalently bonded $A \beta$ aggregate oligomer products. Therefore, $A \beta$-containing ISF must be cleared from the brain parenchyma before oligomer formation. Based on tracer studies, Carare and Weller and their collaborators have suggested $[14,20]$ that the primary route for this $\mathrm{A} \beta$ waste clearance process is primarily along vascular basement membranes. This proposed A $\beta$ ISF efflux clearance route starts at the capillary basement membrane connecting to the basement membranes of the smooth muscle cells of the arteriole and then to the basement membranes of the arteries leading out of the cortex and the meninges arteries in the SAS, ultimately flowing into the lymphatic system. The flow of $A \beta$-containing ISF along this very narrow, tortuous, and relatively tightly confined route offers many opportunities for detrimental laminar and extensional shear-induced $A \beta$ aggregation reactions.

Figure 5 summarizes the above discussion regarding the $A \beta$-containing CSF flow path that parallels the arterial blood flow and illustrates the periarterial return flow ISF suggested by Carare and coworkers $[14,20]$, originating in the brain parenchyma next to the parenchyma capillaries and that flows in the opposite direction from that of the arterial and capillary blood flow, shown in Fig. 5 by dashed lines.

Figure 5 also includes the more recent introduction of another proposed ISF flow pattern, namely the "glymphatic system" introduced by Nedergaard and colleagues [21, 22]. This glymphatic $A \beta$ waste disposal system relies on a proposed tortuous, shearinducing passage through the extracellular spaces
(ECS) in the tightly-packed brain parenchyma, exiting the brain through perivenous flow paths. This glymphatic system is indicated at the center bottom of Fig. 5. The contact between the two Fig. 5 circular brain fluid pathways represents the interface between the vascular CSF and parenchyma extracellular space ISF through the astrocyte OEP openings to the parenchyma.

In the above discussion, the search has been for those regions in the brain where $A \beta$ shear processes might be present. Two main regions have been identified, the vascular basement membranes containing the PVP and the Cararare-Weller ISF exit path, and the brain parenchyma. The next question to be addressed is what happens to $A \beta$ molecules in the latter brain shear region that might initiate an amyloid cascade or other chemical reaction leading to toxic consequences?

\section{Shear effects on amyloid molecules}

Laminar shear (Fig. 2) can originate in any liquids confined to flow between narrow confining walls in the brain vascular system and parenchyma. The conformation of $A \beta$ molecules under shear stress can be distorted in such a way that interior hydrophobic regions of the molecule are exposed to the $A \beta$ 's hydrophilic exterior environment (Fig. 6a, b). Because of the narrowing of the brain fluid passage as it penetrates into the very narrow PVP and ECS flow passages of the vascular system and the brain parenchyma, there is also a high probability 
of developing extensional CSF shear (Fig. 6b). Thus the shearing of the $A \beta$ molecule can cause it to be stretched into more linear, higher energy conformations, designated as $\mathrm{A} \beta *$, the asterisk representing an unstable, shear-excited molecular state.

\section{Protein conformations, wall adsorption, and shear}

When solutions of highly flexible IDP such as $A \beta$ are confined and flow, possibly in a pulsed, oscillating manner, through the brain in narrow CSF or ISF flow passages, such as the very narrow biological confines found in PVP [23] and brain parenchyma, they are probably subjected to significant liquid shear forces that result in reversible $A \beta$ molecular conformation changes. Such shear forces are dependent on a number of factors. Among these are solution flow rate, flow path shape, objects restricting the flow passage around which the fluid must flow within the flow path, and, especially important, cross sectional dimensions that constrict the flow path. For example, liquid shear generated by flow through a capillary is inversely proportional to the cube of its radius under otherwise identical flow conditions! Thus, halving the inner diameter of a capillary increases the shear rate by a factor of eight, given the same flow rate.

In our capillary experiments [3], we observed in electron micrographs a significant protein coating on the capillary wall. There were also other indications of accelerated losses of proteins to the wall during repeated protein injections, suggesting that the initial wall coating promoted increased wall adsorption in a catalytic manner. In these experiments, there were indications of a dependence of the rate of this wall aggregation on initial dissolved protein concentration. There were also indications that metastable wall aggregates slowly dissolved back into the solvent following cessation of the mobile phase solvent flow through the capillary. This combination of reversibility and protein concentration dependence is highly reminiscent of previously proposed mechanisms for in vitro reversible amyloid fibril formation [8] as well as of AD clinical studies showing correlations between increasing dementia with $A \beta$ concentration in brain CSF [1].

As indicated on the left side of Fig. 6, protein conformations in quiescent solutions are, on average, such that hydrophilic amino acid side chains are concentrated on the surface of the protein directly interfacing with the aqueous environment, whereas

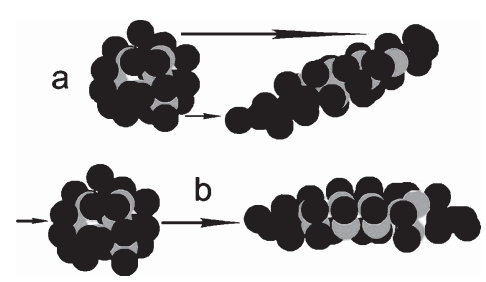

Fig. 6. Hypothetical protein showing gray balls as hydrophobic groups and black balls as hydrophilic groups. The protein in (a) has undergone severe laminar shear to emphasize the resulting increased exposure of hydrophobic groups to the surrounding solvent. The same is true in (b) for extensional shear, where the molecule is essentially pulled lengthwise. Models are purely hypothetical. Energy is added to the molecule in both (a) and (b) forming unstable molecules. Most brain shear events probably have contributions of both (a) and (b) types of shear.

hydrophobic side chains are, on average, found near the interior of the protein. Stretching and distortion of the sheared protein forces inner hydrophobic sites closer to the hydrophilic fluid surface, producing a more surface-hydrophobic molecular conformation. In such a conformation, protein molecules under shear conditions are rendered much more vulnerable to aggregation with other nearby sheared protein molecules. Wall solute adsorption is likely on internal brain regions when membrane surface bonding forces correspond to those of shear-stressed solutes. Stressed proteins are apparently strongly attracted to a stainless steel capillary wall, probably because of polar and ionic attractions. When these stressed proteins adsorb on a surface, the resulting protein coating can change the surface characteristics in such a manner that alters the types of stressed proteins attracted to that surface. Repeated injections without column cleaning gave some indications of a catalytic effect of initial wall coatings. That is, losses to the wall per injection increased with successive injections of the same volume into the same column, in some cases with so much wall adsorbed solute that laminar flow was no longer observed.

If shear is significant in fluid flow within the brain, and if the primary types of shear are laminar and extensional, then the parts of the brain that experience the maximum shear are at the confining flow path walls. That is, at the membrane surfaces confining and restricting ISF and CSF flow rates.

$\mathrm{A} \beta$ is found in CSF entering the brain in PVP surrounding brain arteries. Studies indicate that $A \beta$ containing ISF enters the vascular walls through the capillary basement membranes [14]. It is suggested that there are numerous opportunities in the vascular walls where shear could be responsible for 
the formation of CAA deposits formed within brain artery walls.

The structure of the PVP is currently the subject of much research and uncertainty regarding the structure of this CSF flow pathway [14-16]. Estimates of relative dimensions of the perivascular brain fluid flow path confinement spaces needed for shear rate estimates in the vascular and parenchymal brain regions indicate that parenchyma dimensions are many orders of magnitude smaller than those of the PVP [12]. However, CSF flow rates are probably also many orders of magnitude larger in the PVP system than in the vascular system. Thus it is entirely possible that shear rates could be roughly comparable in the two brain fluid systems. Despite all these uncertainties, it is becoming increasingly clear that neither the $A \beta$ present in the perivascular nor that in the parenchymal fluid system is in a totally quiescent situation, and therefore both are subject to fluid shear that could possibly initiate $A \beta$ amyloid condensation and cascade reactions.

The majority of in vitro experiments with $\mathrm{A} \beta$ and its toxic oligomers have been carried out in quiescent situations. A critical experiment on shear-induced $\mathrm{A} \beta$ aggregation has been carried out under carefully controlled shear conditions [4]. However, the experimental conditions of this and all other shear experiments with $A \beta$ do not tightly confine the liquid and generate the same types of shear that can be imagined within the brain, especially with respect to extensional shear.

\section{Proposed chemical effects of shear on A $\beta$ in ISF and CSF}

It is suggested in this paper that CSF flow within certain sections of the PVP and tunica generated by a systole pulse may be sufficient to create a shearstressed, conformation-altered $\mathrm{A} \beta$ molecule, $\mathrm{A} \beta^{*}$, near the adventitial, muscle cell, and astrocyte feet walls of the PVP. The shear-energy rich $A \beta *$ then preferentially diffuses either toward or away from the walls, thermodynamically driven to diffuse to a lower shear region [19]. Diffusion away from the wall lowers the shear rate and, depending on the inherent lifetime of the $A \beta^{*}$ molecule, it reverts to the original low energy state $A \beta$ conformation, releasing its shear energy to the environment as heat.

An $\mathrm{A} \beta^{*}$ molecule found in a liquid next to a wall subjected to maximum shear stress can be forced into a high-energy, shear-distorted conformation, exposing to the aqueous surroundings new hydrophobic and hydrophilic and possibly ionic regions of the $A \beta$ molecule. With increasingly high $A \beta *$ concentration brain fluids present near the wall, such as those found in $\mathrm{AD}$, collisions between two $\mathrm{A} \beta *$ molecules have an increasing probability of forming a more stable $(A \beta)_{2}$ dimer near the wall because both entities have similar attractive hydrophobic and hydrophilic intermolecular forces attracting nearby $\mathrm{A} \beta *$ molecules to each other. This dimer is more stable because less hydrophobic region is exposed to water and would be expected to lower the excess stress energy and release it during the transition from $\left(A \beta^{*}\right)_{2}$ to $(A \beta)_{2}$ as thermal energy to its surroundings. Formation of either one of these dimers could then initiate an amyloid cascade reaction by reacting with more $A \beta$ or $\mathrm{A} \beta *$ molecules.

It has been proposed that there is rapid fluid exchange at the capillary interface of ISF $[15,16]$, presumably through the astrocyte OEP. Because the width of this OEP opening is on the order of $20-50 \mathrm{~nm}$ [24], there is a probability of shear-induced $A \beta$ conformation change, especially for molecules located next to the OEP wall depending on the flow rate through the OEP. Thus, it is proposed here that this resistance to CSF flow from the PVP through the astrocyte OEP into the parenchyma as well as the resistance to the perivascular ISF return flow results in energetic conformation changes that depend on the ISF flow rates, the degree of flow restriction, and on the ambient $A \beta$ concentration. It is proposed that the ultimate consequence of these restricted tortuous cardiovascular passages is the formation of shear-induced CAA solids within and around the vascular walls resulting from shear-induced $A \beta *$ cascade reactions, one consequence being the possible filling or at least narrowing of the astrocyte OEP flow path.

\section{Flow resistance and $C A A$ deposits from basement membrane components}

Perivascular ISF return flow through the artery or arteriolar walls is reported to be through the basement membranes, primarily those of the muscle cells in the tunica media $[14,25]$. Basement membranes are important not only because of this proposed perivascular ISF return flow and changes in arterial structure caused by AD [26] but also because of their possible importance in potential brain parenchymal shear as discussed above and later in this paper.

There are three major components of basement membranes: laminin, collagen IV, and heparan sulfate 
proteoglycans (HSPG). Laminin and collagen form the major structural elements of the basement membrane. All of these components will interfere with the flow of $A \beta$-containing brain fluids in both vascular and parenchymal regions. HSPGs, one of which is symbolically diagramed in Fig. 7 (laminin and collagen components are not shown), appear to be one of the most chemically active parts of the basement membrane. HSPG and other proteoglycans found in this membrane perform different biological functions. The co-localization of HSPG with several forms of amyloid plaque implies an involvement of this molecule in the mechanism of amyloid formation [26]. Alterations of these membrane components take place during the progression of $\mathrm{AD}$, in particular during the growth and thickening of the vascular wall. With respect to vascular processes in this paper, it is suggested that HSPG molecules may help catalyze the formation of CAA deposits. A postulated mechanism for this catalysis is described in Supplementary Material B. Further discussion of basement membrane processes is found below.

\section{SHEAR WITHIN BRAIN PARENCHYMA}

\section{Fluid flow of ISF in parenchymal extracellular space}

Recent research has supported the idea that parenchymal ISF flow is slow, but not quiescent. One review [15] challenges the traditional animal studiesbased understanding of CSF physiology, citing more recent molecular and cellular biology and NMR neuroimaging reports of complex, poorly understood CSF flow reversals. In addition to a directed flow of CSF within the brain, according to this review, there is a "pulsatile to and fro movement throughout the entire brain." There is, according to Brinker et al. [15], "a continuous bidirectional fluid exchange at the blood brain barrier [that] produces flow rates which exceed the choroidal CSF production rate by far." They conclude that "CSF circulation is much more complex, a combination of directed bulk flow, pulsatile to and fro movement, and continuous bidirectional fluid exchanges at the blood brain barrier and the cell membranes at the borders between CSF and ISF spaces."

Thus, in view of the above comments and if results of the mouse experiments of Xie et al. [27] can be extrapolated to humans, there may be oscillatory ISF flow with little net ISF flow through the human brain parenchyma during wakeful periods. If the above

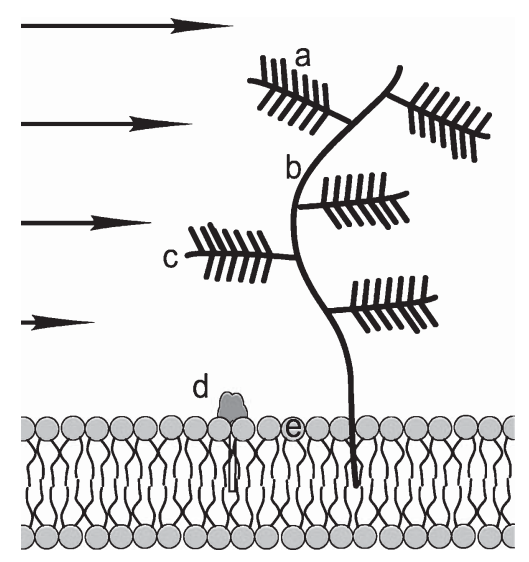

Fig. 7. Heparan sulfate proteoglycan (HSPG) consisting of: a) negatively charged sulfate compound "needles"; b) main branch hyaluronan; c) protein side branch; d) polar head of GM1 ganglioside that is reported to bind to amyloid oligomers: e) polar membrane phospholipid head. Negatively charged HSPG molecules attract positively charged $A \beta$ side chains, if they are not internally bound. It is proposed that in sheared $A \beta^{*}$ they are HSPG accessible. GM1 molecules are rich in hydrophilic $-\mathrm{OH}$ groups that are attractive to $A \beta$ and $A \beta^{*}$ groups. In addition, $\mathrm{HSPG}$ "trees" interfere with ISF flow and presents a flow obstacle. Other molecular flow obstructions such as laminin and collagen are not shown. Arrows indicate differential shear flow near the membrane. Figure not to scale.

is the case, what are the different possible fates of the two major $A \beta$ isoforms following their release from the synapse? Are there possible shear-related hypotheses that can be constructed and tested in laboratory brain parenchyma models?

\section{The unique role of $A \beta_{42}$ in brain parenchyma}

Hong et al. [28] report that $A \beta_{42}$ oligomers in mouse model $\mathrm{AD}$ experiments adsorb strongly to the ganglioside GM1 embedded in mouse hippocampal neuron membranes (see Fig. 7d). Such neuron surface oligomer adsorption is suspected to cause interference with neuron functioning. This $A \beta_{42}$ oligomer adsorption process is reported [28] to account for a significant portion of the missing anticipated free $\mathrm{A} \beta_{42}$ in the parenchymal brain segment, in comparison with the amount estimated to be released from the synapse. Therefore, it is assumed that $A \beta_{40}$, the only major soluble $A \beta$ monomer conformer remaining, is flushed out of the parenchyma and is found in circulating brain CSF and thus predominates in the brain vascular amyloid deposits associated with CAA. Two important questions remain unanswered. Why isn't $A \beta_{40}$ also involved in parenchymal processes? What is the origin of toxic $A \beta_{42}$ oligomers in 

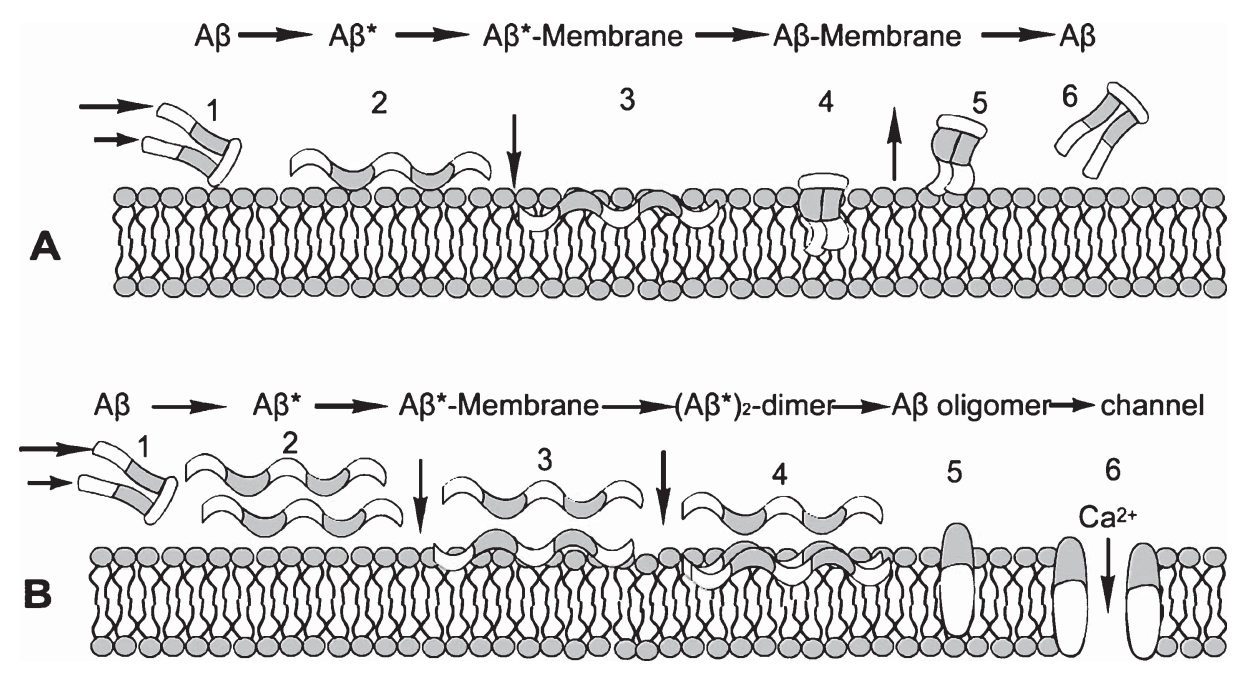

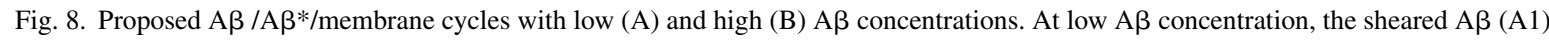
molecule forms $A \beta^{*}(\mathrm{~A} 2)$ is attracted to and adsorbed by the membrane (A3), alters its conformation to a low energy state $A \beta$ (A4) and is released from the membrane (A5) in (A6, reoriented to A1), keeping the steady state $A \beta$ concentration low. At high $A \beta$ concentrations (B), this A cycle is interrupted by the formation of an $A \beta^{*}$ dimer (B3, B4), which initiates the formation of stable oligomers within the membrane (B5). This process can be toxic, for example, by forming an open membrane flow path that allows free passage to ions such as $\mathrm{Ca}^{2+}$ (B6). White segments of the $\mathrm{A} \beta *$ molecule are hydrophobic and gray segments are hydrophilic. The same scheme is applied to the membrane, hydrophobic tails of which attract corresponding $A \beta$ segments, and similarly with hydrophilic segments.

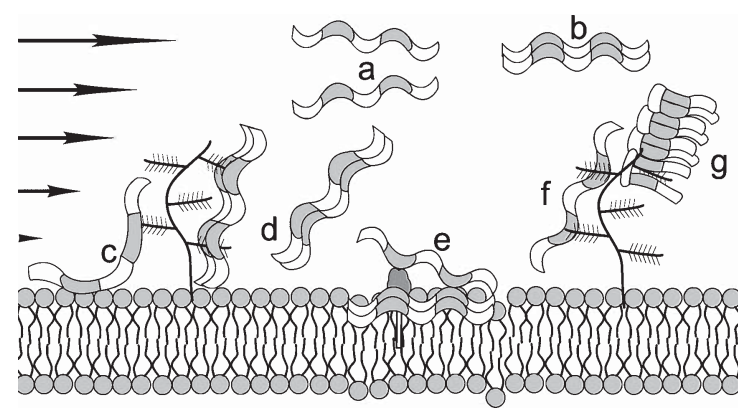

Fig. 9. Flow impediments reacting with $A \beta^{*}$. (a) Sheared $A \beta^{*}$ molecules that are attracted to one another to form an $\mathrm{A} \beta$ dimer (b) without interacting with the wall or flow impediments; (c) single $A \beta^{*}$ attracted to both the membrane and the proteoglycan; (d) $\mathrm{A} \beta *$ dimer that could either be released or form the nucleus for a higher order oligomer that remains attached to this location; (e) GM1, a ganglioside that has a hydrophilic bulky exterior group and a hydrophobic anchor and has immobilized a membrane-embedded $\mathrm{A} \beta$ dimer providing a nucleus for oligomer formation in this location; (f) a single $A \beta^{*}$ locked on one proteoglycan that might then convert to a hairpin conformation that is shown in $(\mathrm{g})$ as a focal point for the formation of a protofibril. This latter process could be similar to that proposed by Metzner et al., for the downstream formation of fibrils in their experiments with commercial polymers (Fig. 4 in ref. [19]). HSPG not to scale.

the parenchyma? Possible answers to these questions are discussed in detail in Supplementary Material C. A short summary covering the major points made in the Supplementary Material follows.

\section{Suggested possible consequences of parenchymal shear}

It is proposed that even very slow ISF parenchymal flow rates can cause shear-induced formation of $A \beta_{42} *$, but generate insufficient shear energy to form $A \beta_{40}$, in the very narrow ECS (extracellular spaces) of the parenchyma. Because the shear is highest next to the membrane surface, it is proposed that there is sufficient shear energy to form the $A \beta_{42}$ * molecules near the membrane that are shear-distorted sufficiently to expose sufficient new potential bonding sites so that they are attracted to corresponding bonding sites on the membrane surface.

It is suggested that while adsorbed to the membrane, the $A \beta_{42} *$ loses its shear energy to the membrane, changes its conformation back to that of $\mathrm{A} \beta$ and ultimately leaves the membrane to again join the flowing ISF. If the local $A \beta$ concentration is sufficiently low, this adsorption/de-excitation/desorption cycle allows both $A \beta_{40}$ and $A \beta_{42}$ to be eliminated from the parenchyma. This cycle merely lowers the steady state ISF concentration of $A \beta_{42}$. However, as local $A \beta$ concentration increases, there is increased probability that, while adsorbed on the membrane, an $\mathrm{A} \beta_{42} *$ is able to encounter another $\mathrm{A} \beta_{42} *$ adsorbed to the membrane surface and form an $\left(\mathrm{A} \beta_{42} *\right)_{2}$ dimer, which loses its energy to the membrane to become a stable membrane-attached $\left(A \beta_{42}\right)_{2}$ dimer. This 


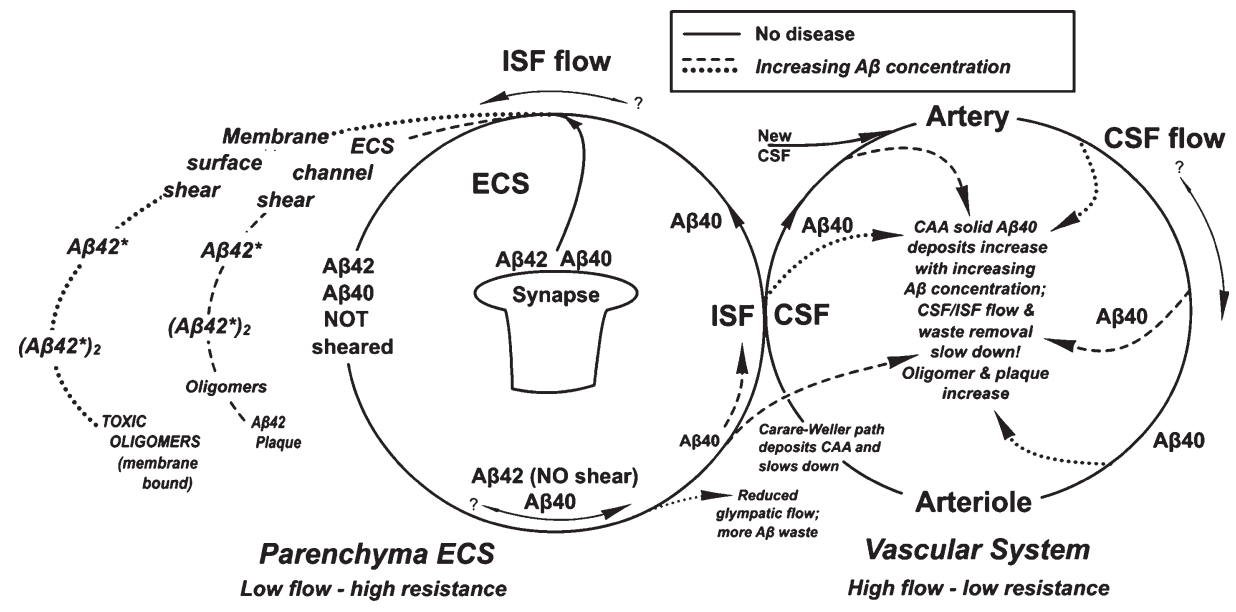

Fig. 10. Summary of the processes described in this paper shows two cycles that merge at the ISF/CSF interface. The solid lines represent the processes without disease. Dashed and dotted lines are those processes occurring with AD. The dashed line represents the "non-wall" lower energy $\mathrm{A} \beta^{*}$ aggregate formation process and the dotted line represents the results of the higher energy A $\beta *$ "wall" shear aggregate product (see text for definitions).

stable dimer can now react with successive $A \beta_{42} *$ molecules to ultimately form neurotoxic oligomers. Except for the initial adsorption of the $A \beta_{42} *$ from the ISF to the membrane, the remaining oligomer formation could take place on the membrane.

Not all the ISF A $\beta$ molecules will be found on or will migrate to the membrane walls confining the flowing ISF. Those $A \beta$ molecules that are just near enough to the membrane to be exposed to slightly less than maximum shear will encounter large molecules in the ECS, some of which jut out from but are anchored to the membrane and some of which are suspended in the ECS. These molecules, collectively designated as the perineuronal network (PNN) [29], will act as ISF flow impediments and cause shear, especially if present at high densities, where this can cause extensional shear. Many of these macromolecular structures contain component proteins which have regions containing bonding sites that can attract passing $A \beta$ molecules. It is proposed that, when the $A \beta$ concentrations are low, shear-induced $\mathrm{A} \beta_{42} *$ molecules are attracted and attach temporarily to these HSPG flow impediments before reversibly detaching after a short period. However, at higher $A \beta$ concentrations, two $A \beta_{42}$ * molecules, one attached to an HSPG, can collide in the ISF stream and condense before the release of the adsorbed $A \beta_{42}{ }^{*}$, and start building a condensation chain that ultimately becomes an $A \beta$ fibril.

Thus, there are postulated to be two separate shear-induced processes, one leading to toxic membrane-embedded oligomers, all shear-induced chemical action taking place on the membrane surface at high $A \beta_{42}$ concentrations, and the other taking place near the membrane on HSPG molecules because of shear-induced chemical interactions taking place because of HSPG flow impediments. Each of these processes is separately highly sensitive to increasing $A \beta$ concentration because of competition between adsorption/desorption and dimer-forming chemical reactions. Symbolic illustrations and more detailed justification for the above summary are found in Supplementary Material C.

There would appear to be a shear-induced coupling between the vascular and parenchymal flow cycles illustrated in Fig. 10. Both of these cycles are highly sensitive to increases in $A \beta$ concentration. However, they involve different $A \beta$ isoforms. $\mathrm{A} \beta_{42}$ is the key isoform involved in both the membrane and ESC/PNN region reactions involving the removal via membrane oligomer and fibril formation of most of the $A \beta_{42}$ molecules from the parenchymal ISF. Therefore, any $A \beta$ that is cleared from the parenchyma through the proposed perivascular ISF Carare-Weller return and glymphatic ISF A $\beta$ clearance pathways involves primarily the $A \beta_{40}$ isoform, which is that found predominantly in CAA deposits as seen in Fig. 10. The dashed and dotted lines in Fig. 10 illustrate the main hypotheses contained in this paper.

\section{SHEAR IN OTHER AMYLOID DISEASES}

The above hypotheses regarding shear-induced $A \beta$ conformation changes possibly involved in $\mathrm{AD}$ need 
not be limited to $A \beta$ or to this disease. A similar case as that outlined above can be made for the importance of investigating the effects of shear on microtubule associated tau protein molecules, which are also in the IDP protein class and have neurotoxic oligomers [30]. This author strongly suggests that fundamental in vitro research on the effects of shear on amyloids and tau molecules, especially the interactions among amyloids, tau, shear, and membranes, deserves a greatly increased $\mathrm{AD}$ research effort.

These shear-based chemical mechanism suggestions could well be applicable to many other amyloid-synuclein related diseases. Prions are also intrinsically disordered proteins, have many different strains with the same overall molecular composition, each strain representing a different conformation [31]. Two different strains of $\alpha$-synuclein, another intrinsically disordered protein, with two different structural conformations have been reported to cause two different neurodegenerative disorders [32], Parkinson's disease and multiple system atrophy. Could these and other disease-causing, amyloid strains arise from different degrees and types of shear in different parts of the brain or body?

\section{SUMMARY}

This paper explores the potential regions of the brain where laminar and extensional liquid shear might be present. On the basis of published liquid shear literature involving $A \beta$ and other protein solutions, the author proposes that liquid shear induces distorted-conformation excited molecular states $A \beta^{*}$ that have been previously identified as "unfolded" or "misfolded" proteins that initiate amyloid cascade reactions. It is proposed that these higher than thermal energy $A \beta^{*}$ states are produced in CSF flowing next to or very near the fluid-confining walls of the brain CSF perivascular system and in interstitial fluid flowing more slowly through the tightly confined extracellular parenchymal spaces. These various shear energy-excited states are suggested as possible initiating molecular agents for membrane toxic oligomers, plaque formation, and CAA in a complex shear-related interplay between vascular and parenchymal $A \beta$ cycles that are both highly dependent on $A \beta$ concentration in a non-linear manner. It is suggested that the current research focus on identifying and studying toxic soluble oligomers of $A \beta$ present in quiescent solutions may be incomplete and possibly even misleading. Several papers in the literature $[7,8]$ imply amyloid cascade products might differ in quiescent and flow conditions in which $A \beta$ is sheared. This paper suggests that the brain membrane may be necessary to form a critical type of neurotoxic oligomer formed in an amyloid cascade. If the hypotheses put forward in this paper are correct, it is suggested that researchers should also be focusing on eliminating or preventing the formation of $\mathrm{A} \beta_{42} *$ and the $\mathrm{A} \beta_{42} *$-membrane complex, since these two entities are suggested as precursors to toxic membrane oligomers. The $A \beta_{40} *$ is proposed as the precursor to CAA vascular solids suggested as the cause of reduced clearance rates of parenchymal $\mathrm{A} \beta$.

\section{Suggested experimental tests of the hypotheses contained in this paper}

There are many unknowns regarding human brain fluid flow patterns and resulting shear rates. However, most of the shear hypotheses presented in this paper are testable in the laboratory. Many previous amyloid shear studies have been carried out using comparatively irreproducible methods such as stirring and shaking or are concerned with processes at the airwater surface, which are important industrially. The brain presumably does not host such surfaces. Those few purely liquid experimental amyloid studies carried out with reproducible liquid shear forces do not have confined space geometries or dimensions that are suitable models for the kind of shear processes that possibly occur within the brain, especially with respect to extensional shear.

Investigations are proposed using a different approach to shear experiments. The author will be submitting another closely related paper to this one that proposes a new, but simple basic experimental technique involving capillary tubing and other flow restrictive geometries that generate laminar and/or extensional shear and that is designed for testing many of the amyloid shear hypotheses proposed in this paper. Numerous critical experiments using this novel technique will be suggested in that paper for testing these hypotheses. The technique is based upon that used in our early shear experiments, but involves new experimental innovations.

This retired researcher urges others to explore this new, potentially groundbreaking and, it is believed, critically important research area. 


\section{ACKNOWLEDGMENTS}

The author gratefully acknowledges helpful discussions and communications with Jeffrey Iliff, Lila Gierasch, Richard Vachet, Herbert Blades, Roy O. Weller, David Teplow, Dave Dunstan, David Talaga, Lorna Alston, Robert Thorne, David Colby, Conrad Yocum, Maxwell Siegel, Richard Kline, Susan Trumbore, and Virginia Ahrens.

The author's disclosure is available online (http:// $\mathrm{j}$-alz.com/manuscript-disclosures/16-0027r3).

\section{SUPPLEMENTARY MATERIAL}

The supplementary information is available in the electronic version of this article: http://dx.doi. org/10.3233/JAD-160027.

\section{REFERENCES}

[1] Johansson P, Mattsson N, Hansson O, Wallin A, Johansson $\mathrm{J}-\mathrm{O}$, Andreasson U, Zetterberg H, Blennow K, Svensson J (2011) Cerebrospinal fluid biomarkers for Alzheimer's disease: Diagnostic performance in a homogeneous monocenter population. J Alzheimers Dis 24, 537-546.

[2] Blennow K, Zetterberg H (2015) The past and the future of Alzheimer's disease CSF biomarkers-a journey toward validated biochemical tests covering the whole spectrum of molecular events. Front Neurosci 9, 345.

[3] Trumbore CN, Tremblay R, Penrose J, Mercer M, Kelleher F (1983) Unusual flow behavior in high-performance liquid chromatography capillary tubing. J Chromatogr $\mathbf{2 8 0}$, 43-57.

[4] Dunstan DE, Hamilton-Brown P, Asimakis P, Ducker W, Bertolini J (2009) Shear flow promotes amyloid- $\beta$ fibrilization. Protein Eng Des Sel 22, 741-746.

[5] Beckard I, Asimakis P, Bertolini J, Dunstan D (2011) The effects of shear flow on protein structure and function. Biopolymers 95, 733-745.

[6] Knowles TPJ, Vendruscolo M, Dodson CM (2014) The amyloid state and its association with protein misfolding diseases. Nat Rev Mol Cell Biol 15, 384-397.

[7] Qiang W, Kelley K, Tycko R (2013) Polymorph-specific kinetics and thermodynamics of $\beta$-amyloid fibril growth. $J$ Am Chem Soc 135, 6860-6871.

[8] Cohen SIA, Linse S, Luheshi LM, Hellstrand E, White A, Rajah L, Otzen DE, Vendruscolo M, Dobson CM, Knowles TPJ (2013) Proliferation of amyloid- $\beta 42$ aggregates occurs through a secondary nucleation mechanism. Proc Natl Acad Sci U S A 110, 9758-9763.

[9] Hayden E, Teplow D (2013) Biophysical characterization of a beta assembly. Alzheimers Res Ther 5, 60 .

[10] De Strooper B, Karran E (2016) The cellular phase of Alzheimer's disease. Cell 164, 603-615.

[11] Meisl G, Yangb X, Hellstrand E, Frohm B, Kirkegaard J, Cohen S, Dobson, C, Linse S, Knowles T (2014) Differences in nucleation behavior underlie the contrasting aggregation kinetics of the $\mathrm{A} \beta 40$ and $\mathrm{A} \beta 42$ peptides. Proc Natl Acad Sci U S A 111, 9384-9389.
[12] Wolak, DJ, Thorne RG (2013) Diffusion of macromolecules in the brain: Implications for drug delivery. Mol Pharmacol 10,1492-1504.

[13] Szentistvanyi I, Patlak CS, Ellis RA, Cserr HF (1984) Drainage of interstitial fluid from different regions of rat brain. Am J Physiol 246, F835-F844.

[14] Morris AWJ, Sharp MM, Albargothy NJ, Fernandez R, Hawkes CA, Verma A, Weller RO, Carare RO (2016) Vascular basement membranes as pathways for the passage of fluid into and out of the brain. Acta Neuopathol 131, 725-736.

[15] Brinker T, Stopa E, Morrison J, Klinge P (2014) A new look at cerebrospinal fluid circulation. Fluids Barriers CNS 11, 10. http://www.fluidsbarrierscns.com/content/11/1/10

[16] Hladky, S., M. Barrand, (2014) Mechanism of fluid movement into, through and out of the brain: Evaluation of the evidence. Fluids Barriers CNS 11, 26. http://www.fluidsbar rierscns.com/content/11/1/26

[17] Dreha-Kulaczewski S, Joseph AA, Merboldt K-D, Ludwig HC, Gärtner J, Frahm J (2015) Inspiration is the major regulator of human CSF flow. $J$ Neurosci 35, 24852491.

[18] Martinez-Lemus, LA (2011) The dynamic structure of arterioles. Basic Clin Pharmacol Toxicol 110, 5-11.

[19] Metzner AB, Cohen Y, Rangel-Nafaile C (1979) Inhomogeneous flows of non-newtonian fluids: Generation of spatial concentration gradients. J Nonnewton Fluid Mech 5, 449462.

[20] Weller RO, Subash M, Preston SD, Mazanti I, Carare RO (2008) Perivascular drainage of amyloid-beta peptides from the brain and its failure cerebral amyloid angiopathy and Alzheimer's disease. Brain Pathol 18, 253-266.

[21] Nedergaard M (2013) Garbage truck of the brain. Science 340, 1529-1530.

[22] Iliff JJ, Lee H, Yu M, Feng T, Logan J, Nedergaard M, Benveniste H (2013) Brain-wide pathway for waste clearance captured by contrast-enhanced MRI. J Clin Invest 123, 1299-1309.

[23] Papisov M, Belov VV, Gannon K, Kimberly S (2013) Physiology of the intrathecal bolus: The leptomeningeal route for macromolecule and particle delivery to CNS. Mol Pharm 10, $1522-1532$.

[24] Iliff JJ, Wang M, Zeppenfeld DM, Venkataraman A, Plog BA, Liao Y, Deane R, Nedergaard M (2013) Cerebral arterial pulsation drives perivascular CSF-interstitial fluid exchange in the murine brain. J Neurosci 33, 18190-18199.

[25] Weller RO, Preston SD, Subash M, Carare RO (2009) Cerebral amyloid angiopathy in the aetiology and immunotherapy of Alzheimer disease. Alzheimers Res Ther 1,6 .

[26] Perlmutter LS, Chu HC (1990) Microangiopathy, the vascular basement membrane and Alzheimer's disease: A review. Brain Res Bull 24, 677-686.

[27] Xie L, Kang H, Kang H, Xu Q, Chen MJ, Liao Y, Thiyagarajan M, O'Donnell J, Christensen DJ, Nicholson C, Iliff JJ, Takano T, Deane R, Nedergaard M (2013) Sleep drives metabolite clearance from the adult brain. Science 342, 373377.

[28] Hong S, Ostaszewski B, Yang T, O’Malley TT, Jin M, Yanagisawa K, Li S, Bartels T, Selkoe DJ (2014) Soluble $\mathrm{A} \beta$ oligomers are rapidly sequestered from brain ISF in vivo and bind GM1 ganglioside on cellular membranes. Neuron 82, 308-319.

[29] Bitanihirwea B, Woo TU (2014) Perineuronal nets and schizophrenia: The importance of neuronal coatings. $\mathrm{Neu}$ rosci Biobehav Rev 45, 85-99. 
[30] Hawkins B, Krishnamurthy S, Castillo-Carranza DL, et al. (2013) Rapid accumulation of endogenous tau oligomers in a rat model of traumatic brain injury. $J$ Biol Chem $\mathbf{2 8 8}$, 17042-17050.

[31] Colby D, Prusiner S (2011) De novo generation of prion strains. Nat Rev Microbiol 9, 771-777.
[32] Peelaerts W, Bousset L, Van der Perren A, Moskalyuk A, Pulizzi R, Giugliano M, Van den Haute C, Melki R, Baekelandt V (2015) $\alpha$-Synuclein strains cause distinct syncleinopathies after local and systemic administration. Nature 522, 340-344. 